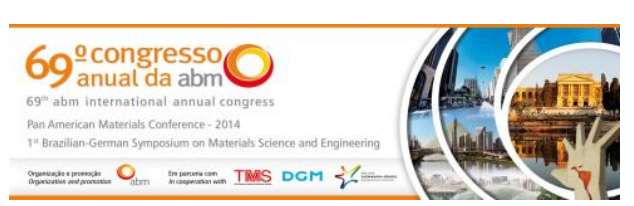

Tema: Diagramas de fases e Transformações de fases

\title{
EFEITO DA ADIÇÃO DE METANO E ARGÔNIO NA REDUÇÃO POR PLASMA DA HEMATITA DE ALTA PUREZA*
}

\author{
Tiago Bristt Gonoring ${ }^{1}$ \\ Adonias Ribeiro Franco Júnior ${ }^{2}$ \\ Estéfano Aparecido Vieira ${ }^{2}$ \\ Ramiro da Conceição do Nascimento Júnior ${ }^{2}$
}

Resumo

Neste trabalho foi avaliado o efeito da adição de metano e argônio sobre a redução a plasma de pós de hematita de alta pureza (com \% $\mathrm{Fe}_{2} \mathrm{O}_{3}>99,00$ e tamanho de partículas $<5 \mu \mathrm{m})$. Os experimentos de redução foram realizados em reator a plasma pulsado $\mathrm{DC}$, nas temperaturas de 360 e $400^{\circ} \mathrm{C}$, por tempos de $30,60,90$ e 120 minutos, sob atmosferas de $100 \% \mathrm{H}_{2}, 2,5 \% \mathrm{Ar}+97,5 \% \mathrm{H}_{2}$ e $2,5 \% \mathrm{CH}_{4}+97,5 \% \mathrm{H}_{2}$. Os resultados mostram que o plasma de hidrogênio puro permite obter frações de redução de até $93 \%$ após por 120 min na temperatura de $400^{\circ} \mathrm{C}$. Sob essas mesmas condições de referência, verificou-se que a adição de argônio, além de garantir uma melhor estabilidade ao plasma, possibilitou a redução de $91 \%$ da hematita. A adição de metano, além de prejudicar a redução, causou instabilidade no plasma. Em todas as condições estudadas, verificou-se que a redução de hematita a ferro se deu em duas etapas: $\mathrm{Fe}_{2} \mathrm{O}_{3} \rightarrow \mathrm{Fe}_{3} \mathrm{O}_{4} \rightarrow \mathrm{Fe}$.

Palavras-chave: Redução; Plasma de hidrogênio; Hematita; Argônio; Metano.

\section{EFFECT OF ADDITION OF METHANE AND ARGON IN THE REDUCTION UNDER PLASMA OF THE HIGH PURITY HEMATITE}

\section{Abstract}

In this work, the effect of both methane and argon on the plasma reduction of high purity hematite powders $\left(\mathrm{Fe}_{2} \mathrm{O}_{3}\right.$ with $>99,00 \%$ and particle size $\left.<5 \mathrm{~mm}\right)$ was evaluated. The reduction experiments were performed in a DC pulsed plasma reactor, at temperatures of 360 and $400^{\circ} \mathrm{C}$, for reduction times of $30,60,90$ and 120 min., using reduction atmospheres containing $100 \% \mathrm{H}_{2}, 2.5 \% \mathrm{Ar}+97.5 \% \mathrm{H}_{2}$ and $2.5 \% \mathrm{CH}_{4}+97.5 \% \mathrm{H}_{2}$. The results show that the reduction carried out with pure hydrogen plasma allows obtaining reduction up to $93 \%$ after 120 min at $400^{\circ} \mathrm{C}$. Under the same conditions, it was found that the addition of argon, not only provided a better stability to the plasma, but also resulted in a reduction fraction of $91 \%$. Reduction experiments with methane-containing atmospheres resulted in both low reduction fraction and plasma instability. In all studied conditions, it has been found that the reduction process occurs in two steps: $\mathrm{Fe}_{2} \mathrm{O}_{3} \rightarrow \mathrm{Fe}_{3} \mathrm{O}_{4} \rightarrow \mathrm{Fe}$.

Keywords: Reduction; Hydrogen plasma; Hematite; Argon; Methane.

1 Engenheiro Metalurgista, Mestrando em Engenharia Metalúrgica e de Materiais, IFES, Campus Vitória, Vitória, ES, Brasil; tiagobristt@gmail.com.

2 Professor Doutor, Programa de Pós Graduação em Engenharia Metalúrgica e de Materiais (Propemm), IFES, Campus Vitória, Vitória, ES, Brasil..

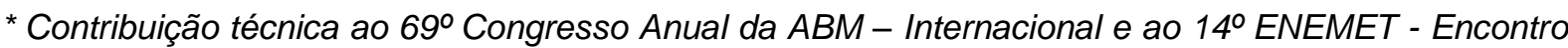
Nacional de Estudantes de Engenharia Metalúrgica, de Materiais e de Minas, 21 a 25 de julho de 2014, São Paulo, SP, Brasil.
} 


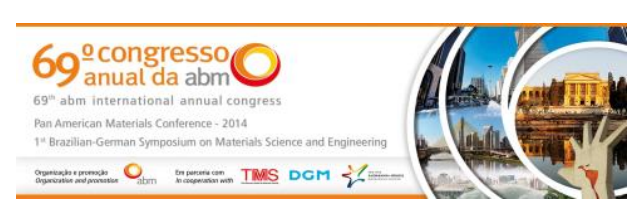

\section{INTRODUÇÃO}

O carbono é o principal combustível e agente redutor utilizado nas indústrias siderúrgicas. A indústria do aço é considerada uma das principais fontes geradores do $\mathrm{CO}_{2}$, principal gás responsável pelo efeito estufa. Do total de aço produzido no mundo, 95\% é através da redução dos minérios de ferro via Alto Forno e cerca de $5 \%$ via redução direta e forno elétrico a arco [1].

As exigências na diminuição das emissões de gases do efeito estufa, principalmente o $\mathrm{CO}_{2}$, aliadas à futura escassez de carvão mineral coqueificável, têm impulsionado a pesquisa por processos alternativos de produção de aço. Por isso, é crescente o esforço para o desenvolvimento de processos em que se usa o gás hidrogênio $\left(\mathrm{H}_{2}(\mathrm{~g})\right)$ como agente redutor. $O$ hidrogênio é um excelente agente redutor em temperaturas elevadas e o subproduto de sua reação com o óxido de ferro ou qualquer outro óxido é o vapor d'água $[2,3]$.

Quando o gás hidrogênio $\left(\mathrm{H}_{2(\mathrm{~g})}\right)$ passa para o estado de plasma de não equilíbrio, ou plasma frio, além das moléculas de hidrogênio, estão presentes várias outras espécies, como moléculas de hidrogênio energeticamente excitadas $\left(\mathrm{H}_{2}{ }^{*}\right)$, hidrogênio atômico $(\mathrm{H})$, hidrogênio iônico $\left(\mathrm{H}^{+}\right)$, fótons e elétrons livres.

Diferentemente do hidrogênio molecular $\left(\mathrm{H}_{2}\right)$, as espécies $\mathrm{H}$ e $\mathrm{H}^{+}$são capazes de reduzir termodinamicamente vários óxidos metálicos, tanto em baixa quanto em alta temperatura, conforme mostra a Figura $1[4,5]$.

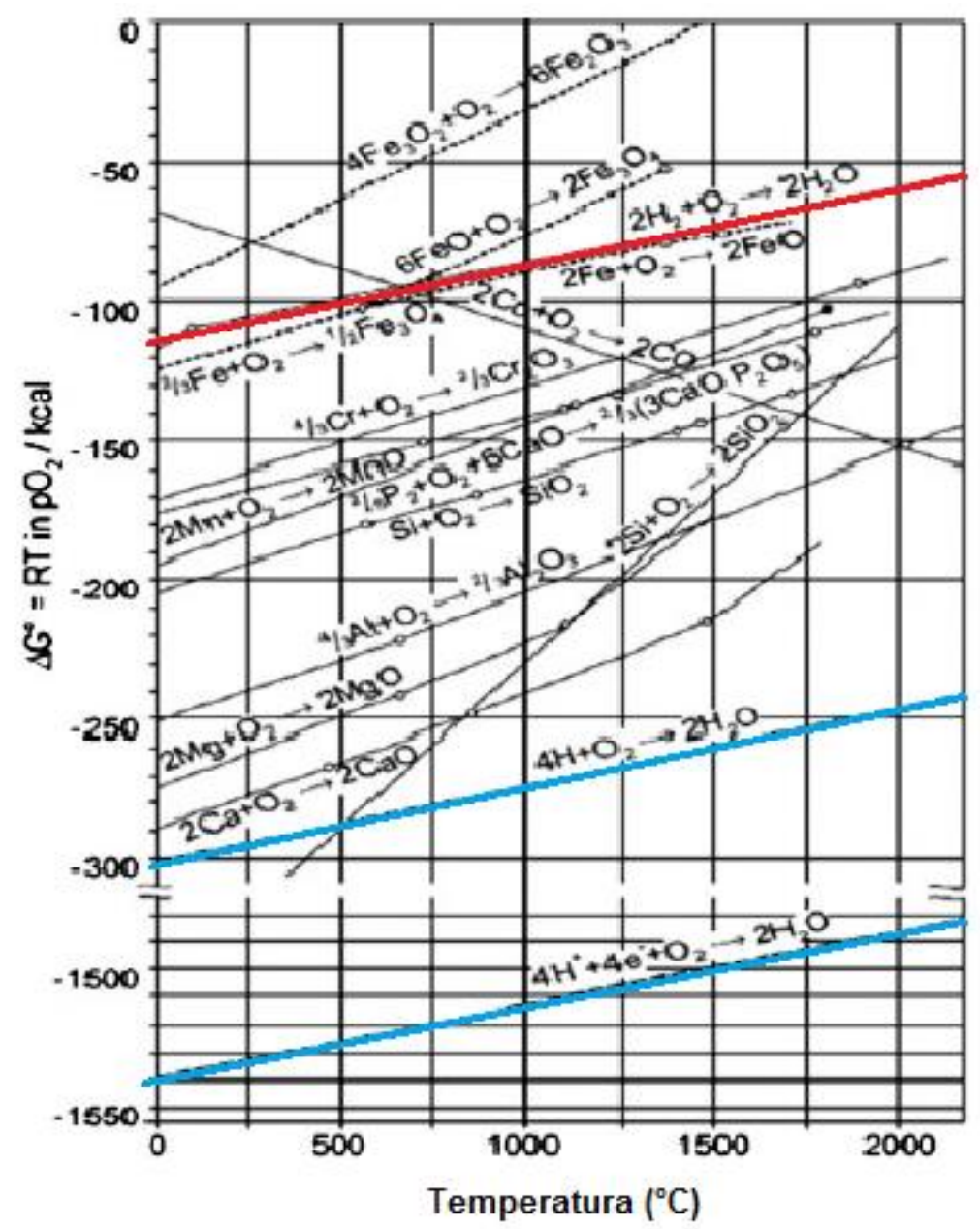

Figura 1. Diagrama de Ellingham Richardson [4].

* Contribuição técnica ao $69^{\circ}$ Congresso Anual da ABM - Internacional e ao 14ํㅡㄹ ENEMET - Encontro Nacional de Estudantes de Engenharia Metalúrgica, de Materiais e de Minas, 21 a 25 de julho de 2014, São Paulo, SP, Brasil. 
Como pode ser visto no diagrama de Ellingham-Richardson da Figura 1, 0 hidrogênio molecular $\left(\mathrm{H}_{2}\right)$ reduz facilmente $\mathrm{Fe}_{2} \mathrm{O}_{3}$ e $\mathrm{Fe}_{3} \mathrm{O}_{4}$, porém o valor do $\Delta \mathrm{G}^{\circ}$ dos equilíbrios $2 \mathrm{H}_{2}+\mathrm{O}_{2}=2 \mathrm{H}_{2} \mathrm{O}$ e $2 \mathrm{Fe}+\mathrm{O}_{2}=\mathrm{FeO}$ são bem próximos. Entretanto, os valores de $\Delta \mathrm{G}^{\circ}$ envolvendo hidrogênio atômico e iônico são muito elevados e negativos em comparação com o do hidrogênio molecular e de outros óxidos. Isso significa que as espécies $\mathrm{H}$ e $\mathrm{H}^{+}$são capazes de reduzir termodinamicamente praticamente a todos os óxidos apresentados no diagrama, tanto em altas quanto em baixas temperaturas. Em razão de no estado de plasma coexistirem ambas as espécies, estão em curso estudos para produzir ferro utilizando o plasma frio de hidrogênio ou o plasma formado a partir de misturas de gases $[5,6]$.

Hibler e Plaul [4] obtiveram ferro reduzido a partir de minério de ferro usando uma tocha de plasma de hidrogênio. Os trabalhos permitiram a concepção de uma planta industrial para a produção de aço baseado no uso do plasma térmico de hidrogênio. Rajput et al [5] realizaram ensaios de redução de minérios de ferro por plasma de hidrogênio em um reator a plasma de micro-ondas com alimentação de $3000 \mathrm{~W}$ de $2,95 \times 10^{9} \mathrm{~Hz}$, pressão de $2,63 \times 10^{3} \mathrm{~Pa}$ e fluxo de $\mathrm{H}_{2}$ de $200 \mathrm{~cm}^{3} / \mathrm{min}$. Segundo os autores, o equipamento em funcionamento garante uma concentração de um átomo de hidrogênio a cada 10 mil moléculas de hidrogênio e 1 íon de hidrogênio a cada 10 mil átomos de hidrogênio. A partir de minério de ferro, com o uso do plasma de hidrogênio eles conseguiram obter uma fração de redução de $94 \%$ a $300^{\circ} \mathrm{C}$ por tempo de 120 minutos, contra apenas $2 \%$ quando se usa o gás de hidrogênio nas

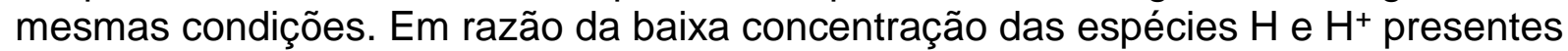
no plasma, eles atribuíram a redução principalmente às moléculas de hidrogênio vibracionalmente excitadas $\left(\mathrm{H}_{2}{ }^{*}\right)$ presentes em altas concentrações [5].

Segundo Ogorodnikova [7], para a molécula de hidrogênio reagir na superfície de um sólido, antes é necessário que ocorra a sua dissociação termicamente ativada.

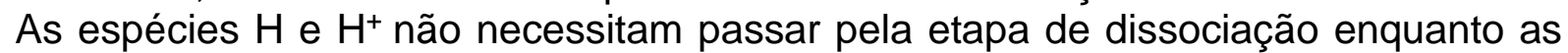
moléculas de $\mathrm{H}_{2}{ }^{*}$ precisam de menor energia de ativação para dissociação. Logo, as espécies ativadas do plasma possuem uma cinética de reação sólido-gás mais eficiente e favorável.

A reação de redução necessita de uma menor energia de ativação quando o hidrogênio passa para níveis de maior energia, ou seja, com a sua conversão em espécies mais energéticas. Assim, através da geração do plasma em temperaturas mais baixas pode-se alcançar um grau de redução com maior eficiência termodinâmica e cinética [8].

Khina et al [9] realizaram experimentos de redução de minério de ferro com tocha de plasma constituído de $25 \%$ de metano $\left(\mathrm{CH}_{4}\right)$ e 75 \% de argônio (Ar) e compararam os resultados obtidos com os de Hibler e Plaul. Os melhores graus de redução foram obtidos com a tocha de plasma composta pela mistura de gases. Os autores justificam que $\mathrm{O}^{\mathrm{CH}_{4}}$ no estado de plasma favorece a geração de espécies que posteriormente reagem com o óxido, gerando o monóxido de carbono (CO) que juntamente com outras espécies presentes no plasma $\left(\mathrm{H}_{2}{ }^{*}, \mathrm{H}_{\left.\text {e } \mathrm{H}^{+}\right)}\right.$são responsáveis pelas altas taxas de redução.

Até a presente data, na literatura não foram encontrados estudos referentes à redução de óxidos usando como agente redutor o plasma frio constituído de misturas de gases $\left(\mathrm{H}_{2}+\mathrm{CH}_{4}\right.$ ou $\left.\mathrm{H}_{2}+\mathrm{Ar}\right)$ e gerado por descargas pulsadas DC.

O objetivo do presente trabalho é comparar a redução da hematita de alta pureza, sob plasma de hidrogênio e gás hidrogênio em um reator a plasma pulsado DC e em seguida, estudar o efeito da adição de metano $\left(\mathrm{CH}_{4}\right)$ e argônio $(\mathrm{Ar})$ na redução a plasma.

\footnotetext{
* Contribuição técnica ao $69^{\circ}$ Congresso Anual da ABM - Internacional e ao 14ํㅡㄹ ENEMET - Encontro Nacional de Estudantes de Engenharia Metalúrgica, de Materiais e de Minas, 21 a 25 de julho de 2014, São Paulo, SP, Brasil.
} 


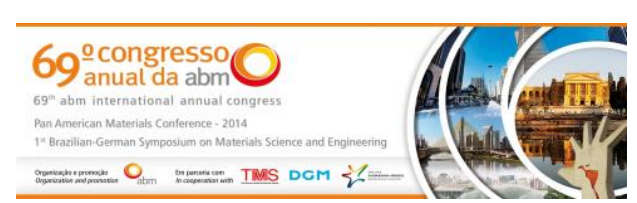

\section{MATERIAIS E MÉTODOS}

A hematita utilizada nesta pesquisa foi fornecida pela Sigma-Aldrich. De acordo com as especificações, o seu grau de pureza é de $99,0 \%$ de $\mathrm{Fe}_{2} \mathrm{O}_{3}$ e $95 \%$ das partículas de pó apresentam granulometria abaixo de $5 \mu \mathrm{m}$.

Tanto os experimentos de redução a gás quanto os a plasma foram realizados em reator a plasma pulsado DC modelo Thor NP da marca SDS, pertencente ao Laboratório de Redução do Instituo Federal do Espírito Santo (IFES), campus Vitória. Um desenho esquemático do reator é apresentado na Figura 2.

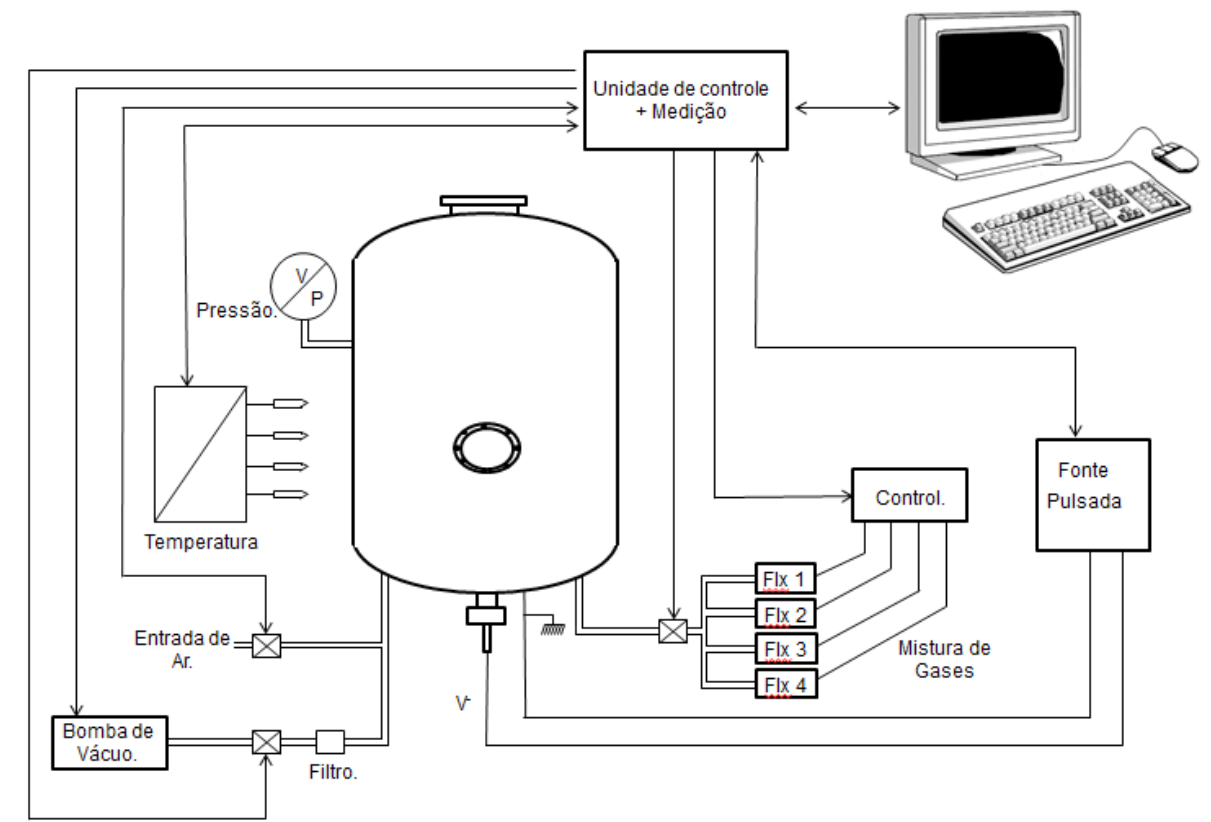

Figura 2. Representação esquemática do reator a plasma pulsado $D C$ utilizado nos experimentos de redução a plasma e a gás.

A câmara do reator é cilíndrica fabricada com aço inoxidável austenítico AISI 304, cujas dimensões são de $500 \mathrm{~mm}$ de diâmetro e $750 \mathrm{~mm}$ de altura. A fonte de tensão pulsada fornece tensão máxima de $650 \mathrm{~V}$, frequência de pulso de $3,4 \mathrm{kHz}$ e período de pulso $(\tau)$ de $250 \mu \mathrm{s}$. A bomba mecânica de palhetas de duplo estágio permite pressão de até $1 \mathrm{~Pa}$ no interior da câmara de vácuo. Há três fluxímetros de gás de vazão máxima de 1000, 750 e $500 \mathrm{~cm}^{3} / \mathrm{min}$. O reator ainda contém medidor de pressão e termopares para o monitoramento da temperatura das amostras Na parte central da câmara do reator, o porta-amostras funciona como catodo (-), enquanto as paredes da câmara funcionam como o anodo (+).

Uma vez evacuada a câmara, introduz-se e mantem a baixa pressão o gás a partir do qual é gerado o plasma, então, quando a fonte de tensão pulsada alimenta o reator, a diferença de potencial faz com que elétrons sejam acelerados do catodo (-) em direção ao anodo (+) [10,11]. As colisões inelásticas dos elétrons com as moléculas do gás levam a excitação, quebra de ligações das moléculas e ionização do gás. As colisões que geram excitações das moléculas são seguidas pela deexcitação das moléculas/átomos com emissão de radiação, que são responsáveis pelo brilho da descarga (glow discharge) gerando-se assim o plasma a frio [10,11].

O período de pulso $(\tau)$ da fonte de tensão é variado para gerar o plasma sobre uma faixa de temperaturas. Este período é composto pelo tempo de pulso ligado da fonte

* Contribuição técnica ao 69 Congresso Anual da ABM - Internacional e ao 14ํㅡㄹ ENEMET - Encontro Nacional de Estudantes de Engenharia Metalúrgica, de Materiais e de Minas, 21 a 25 de julho de 2014, São Paulo, SP, Brasil. 


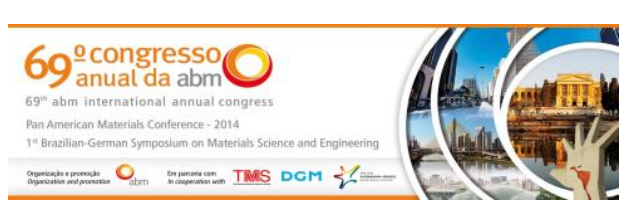

5. Após atingir a temperatura de trabalho, introdução do gás e ajuste da pressão; 6. Após o tempo estipulado para o tratamento, desligamento da resistência do "catodo quente" e fechamento da entrada de gás;

7. Resfriamento sob vácuo até temperatura próxima a ambiente e evacuação (quebra de vácuo).

Após cada um dos experimentos de redução, pesam-se os cadinhos contendo as amostras. A diferença de massa do cadinho contendo a amostra antes e após o ensaio dá o quanto de massa que foi extraída pelo processo de redução. A única possibilidade de perda de massa é a de oxigênio, que sai do reator através da bomba de vácuo na forma de vapor d'água. Então o balanço de massa das amostras para o cálculo do grau de redução $(\alpha)$ é em cima do balanço de massa de oxigênio. Para este calculo utilizamos a equação 1:

$$
\alpha_{i}=\frac{C_{o}^{i}-C_{t}^{i}}{0,99 x 0,30 x m_{0}^{i}}
$$

Em que:

$C_{0}^{i}=$ massa da amostra mais a massa do cadinho (i) antes do ensaio de redução, sendo $\mathrm{i}=1, \ldots, 5$;

$C_{t}^{i}=$ massa da amostra mais o cadinho (i) após a redução, sendo $\mathrm{t}=30,60,90$ e 120 minutos.

$m_{0}^{i}=$ massa da amostra (i) antes do ensaio de redução.

O fator 0,99 é o grau de pureza da hematita e 0,30 representam a \% de oxigênio estequiométrico da hematita $\left(\mathrm{Fe}_{2} \mathrm{O}_{3}\right)$.

$\mathrm{O}$ valor de $\alpha$ determinado em cada experimento corresponde à média aritmética de cinco amostras. Para cada um dos agentes redutores analisados $\left(\mathrm{H}_{2}\right.$ puro, plasma de $\mathrm{H}_{2}$, plasma $\mathrm{H}_{2}+$ Ar e plasma de $\mathrm{H}_{2}+\mathrm{CH}_{4}$ ), de posse dos resultados de $\alpha_{i}(\mathrm{t})$, foram plotadas as curvas de redutibilidade.

A identificação das fases geradas pelos processos de redução foi feita por difração de raios-X (DRX) que permitiu acompanhar a evolução das fases formadas. $O$ equipamento de $D R X$, modelo D2 Phaser Bruker, utilizado para esse fim pertence ao Laboratório de Redução do IFES - campus Vitória. Foi utilizado um tubo com radiação de $\mathrm{Cu}-\mathrm{k}_{a}$ operando em $30 \mathrm{kV}$ e $10 \mathrm{~mA}$. Os padrões de difração foram obtidos com $2 \theta$ variando de $10^{\circ}$ a $100^{\circ}$ e intervalos de $0,02^{\circ}$ por segundo.

\section{RESULTADOS E DISCUSSÃO}

\subsection{Redução de Hematita $\left(\mathrm{Fe}_{2} \mathrm{O}_{3}\right)$ por $\mathrm{H}_{2(\mathrm{~g})}$ nos Estados de Gás e Plasma Frio.}

A Figura 3 apresenta o grau de redução $\alpha=f(t)$ das amostras de hematita após os ensaios de redução por hidrogênio gasoso e plasma frio de hidrogênio nas temperaturas de $360^{\circ} \mathrm{C}$ e $400^{\circ} \mathrm{C}$.

Observa-se que para uma dada temperatura, maiores períodos de tempo favorecem maiores valores de $\alpha$, alternativamente, fixado o tempo maiores temperaturas proporcionam maiores valores de $\alpha$. Ou seja, para os processos de redução aplicados, a redução é diretamente proporcional ao tempo e a temperatura.

De modo semelhante, fixados o tempo e a temperatura, a redução da hematita $\left(\mathrm{Fe}_{2} \mathrm{O}_{3}\right)$ por plasma de hidrogênio foi sempre superior à redução por hidrogênio

\footnotetext{
* Contribuição técnica ao $69^{\circ}$ Congresso Anual da ABM - Internacional e ao 14ํㅡㄹ ENEMET - Encontro Nacional de Estudantes de Engenharia Metalúrgica, de Materiais e de Minas, 21 a 25 de julho de 2014, São Paulo, SP, Brasil.
} 


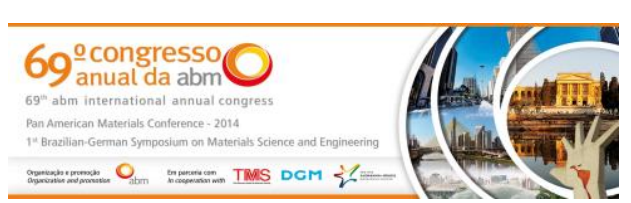

gasoso. A $400^{\circ} \mathrm{C}$ a redução a gás alcançou $57 \%(\alpha \times 100)$ em 120 minutos, enquanto que a plasma, obteve-se $93 \%$ de redução.

Os dados de grau de redução a plasma e a gás a $400^{\circ} \mathrm{C}$ se ajustam com boa aproximação $\left(R^{2}=0,99983\right.$ a plasma; $R^{2}=0,99609$ a gás) a uma curva de uma função sigmoidal. O que indica que a taxa de redução (d $\alpha / d t)$ é crescente até determinado ponto $(\mathrm{t}, \alpha(\mathrm{t}))$ e em seguida é progressivamente decrescente, ou seja, a redução da hematita por plasma e a gás evolui a menores taxas nas etapas finais. Ambas as curvas apresentaram comportamento semelhantes, porém a redução a plasma foi significamente maior. Da mesma forma, a $360^{\circ} \mathrm{C}$ os graus de redução a plasma e a gás tiveram comportamento semelhante até $\mathrm{t}=90$ minutos, a partir daí $\mathrm{a}$ redução a gás tendeu a um valor constante $(\alpha=0,16)$ e a redução a plasma entre $\mathrm{t}=90$ e $\mathrm{t}=120$ minutos apresentou uma elevada taxa de redução alcançando-se um valor de $\alpha=0,54$, valor este bem próximo da redução a gás em $400^{\circ} \mathrm{C}$ e $\mathrm{t}=120 \mathrm{~min}$. e a plasma a $400^{\circ} \mathrm{C} \mathrm{e} \mathrm{t}=60 \mathrm{~min}$. Assim como a $400^{\circ} \mathrm{C}$, a redução a plasma a $360^{\circ} \mathrm{C}$ foi superior a redução a gás.

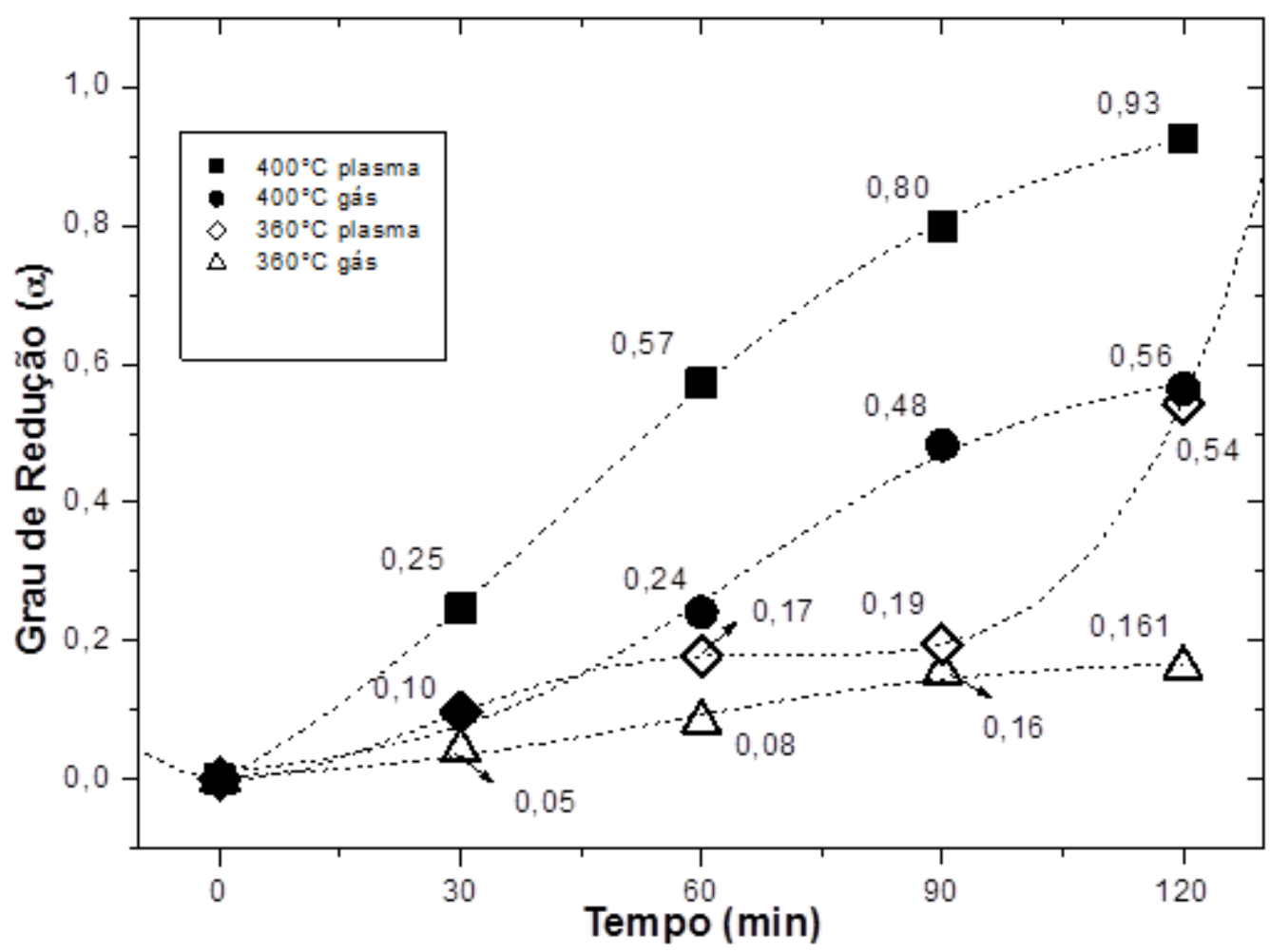

Figura 3. Grau de redução $(\alpha)$ em função do tempo (min.) dos ensaios de redução de hematita por hidrogênio gasoso e plasma frio de hidrogênio.

Os difratogramas apresentados nas Figuras 4(a) e 4(b) são das amostras reduzidas a plasma respectivamente a $360^{\circ} \mathrm{C}\left(60\right.$ e $120 \mathrm{~min}$.) e $400^{\circ} \mathrm{C}(30,60$ e $120 \mathrm{~min}$.). Em todos os difratogramas produzidos das amostras reduzidas a plasma, fizeram-se presente as fases ferro $\left(\mathrm{Fe}_{(\alpha)}\right)$ e magnetita $\left(\mathrm{Fe}_{3} \mathrm{O}_{4}\right)$ e toda o $\mathrm{Fe}_{2} \mathrm{O}_{3}$ foi reduzido. $\mathrm{A}$ diferença básica entre Eles é a intensidade dos picos dos planos de $\mathrm{Fe}_{(\alpha)}$ que estão em função do grau de redução, ou seja, quanto maior o valor de $\alpha$ maiores as intensidades dos picos de $\mathrm{Fe}_{(\alpha)}$ e menor a intensidade dos picos de $\mathrm{Fe}_{3} \mathrm{O}_{4}$. Observase que há grande semelhança entre os difratogramas da Figura 4 (a) em 120 min. $(\alpha=0,54)$ e 4 (b) 60 min. $(\alpha=0,57)$. De fato, a diferença entre a contagem dos picos

\footnotetext{
* Contribuição técnica ao $69^{\circ}$ Congresso Anual da ABM - Internacional e ao 14ํㅡㄹ ENEMET - Encontro Nacional de Estudantes de Engenharia Metalúrgica, de Materiais e de Minas, 21 a 25 de julho de 2014, São Paulo, SP, Brasil.
} 


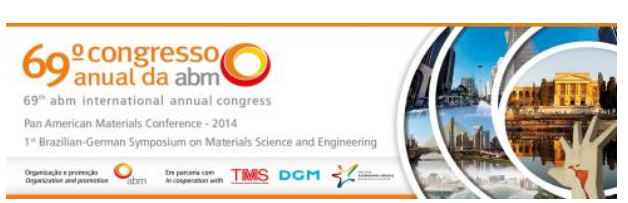

dos planos de ferro, entre estas duas amostras é muito pequena, assim como a dos planos de magnetita.

De posse da evolução das fases apresentadas pelos difratogramas, podemos sugerir que a redução ocorreu em duas etapas: $\mathrm{Fe}_{2} \mathrm{O}_{3} \rightarrow \mathrm{Fe}_{3} \mathrm{O}_{4} \rightarrow \mathrm{Fe}_{(\alpha)}$ ou $\mathrm{Fe}_{2} \mathrm{O}_{3} \rightarrow$ $\mathrm{Fe}_{3} \mathrm{O}_{4}+\mathrm{Fe}_{(\alpha)} \rightarrow \mathrm{Fe}_{(\alpha)}$.

A evolução das fases durante os processos de redução da hematita por hidrogênio gasoso é apresentada na Figura 5 (a) e $5(\mathrm{~b})$. A $360^{\circ} \mathrm{C}$ a hematita não foi completamente reduzida nos tempos de tratamento, e os picos de ferro só foram presente nos tempos de 90 e 120 minutos. Podemos sugerir que a redução ocorreu segundo as etapas: $\mathrm{Fe}_{2} \mathrm{O}_{3} \rightarrow \mathrm{Fe}_{2} \mathrm{O}_{3}+\mathrm{Fe}_{3} \mathrm{O}_{4} \rightarrow \mathrm{Fe}_{2} \mathrm{O}_{3}+\mathrm{Fe}_{3} \mathrm{O}_{4}+\mathrm{Fe}_{(\alpha)}$. Observa-se no difratograma da Figura 5 (b) $t=90$ minutos, a presença somente de magnetita e ferro, enquanto que para $t=120$ minutos há além destas fases uma hematita que não reduziu. Porém a contagem da intensidade dos picos dos planos de ferro na amostra tratada durante $\mathrm{t}=120 \mathrm{~min}$. foram maiores assim como os picos de magnetita menores. Colombo et al [12] realizaram experimentos termogravimétricos de redução de hematita a temperaturas abaixa de $400^{\circ} \mathrm{C}$, segundo os autores é comum a presença de uma hematita não reagida no final da redução quando o grau de redução é pequeno ou não expressivo; e ainda atribuíram esse fenômeno ao surgimento durante a redução de um spinel formado por um ou ambos $\mathrm{Fe}_{3} \mathrm{O}_{4} \mathrm{e}$ $\mathrm{Fe}_{3+\mathrm{w}} \mathrm{O}_{4}$, e proporam o seguinte mecanismo: $\mathrm{Fe}_{2} \mathrm{O}_{3} \rightarrow \mathrm{Fe}_{2} \mathrm{O}_{3}+$ spinel $+\mathrm{Fe}_{(\alpha)}$.
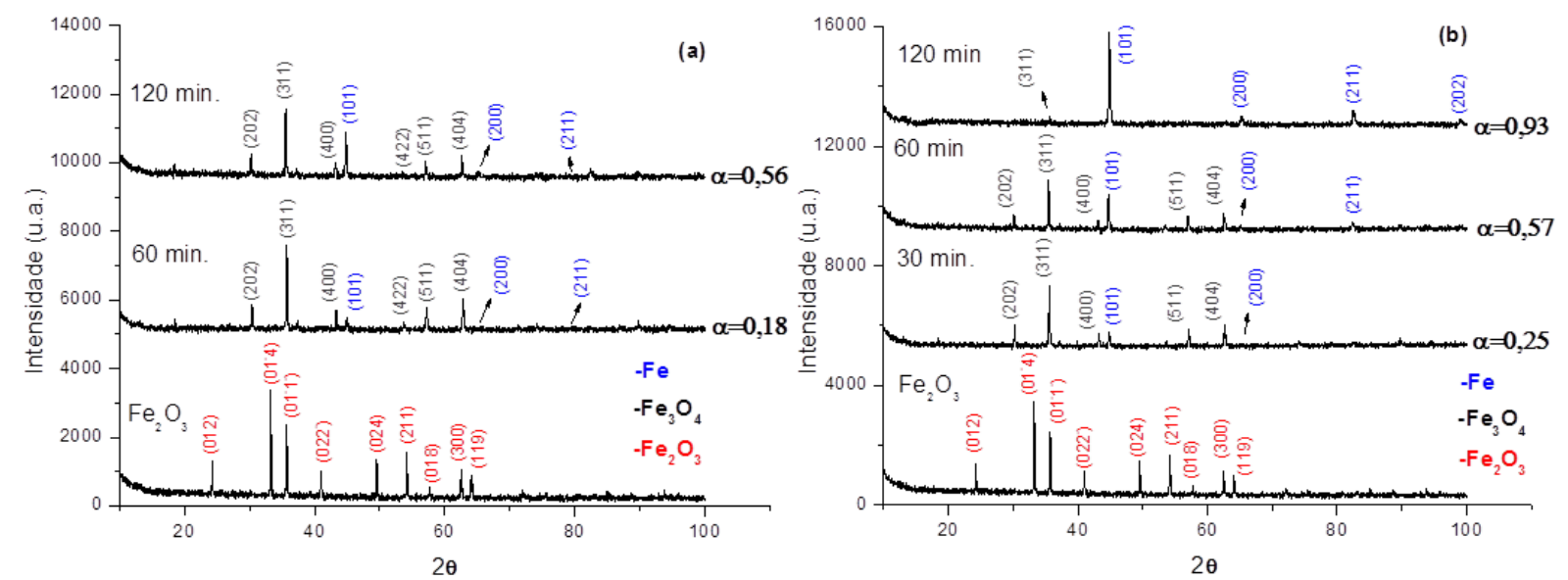

Figura 4. Evolução das fases presentes nos pós de hematita na redução por plasma de hidrogênio, a $360^{\circ} \mathrm{C}$ (a) e $400^{\circ} \mathrm{C}(\mathrm{b})$
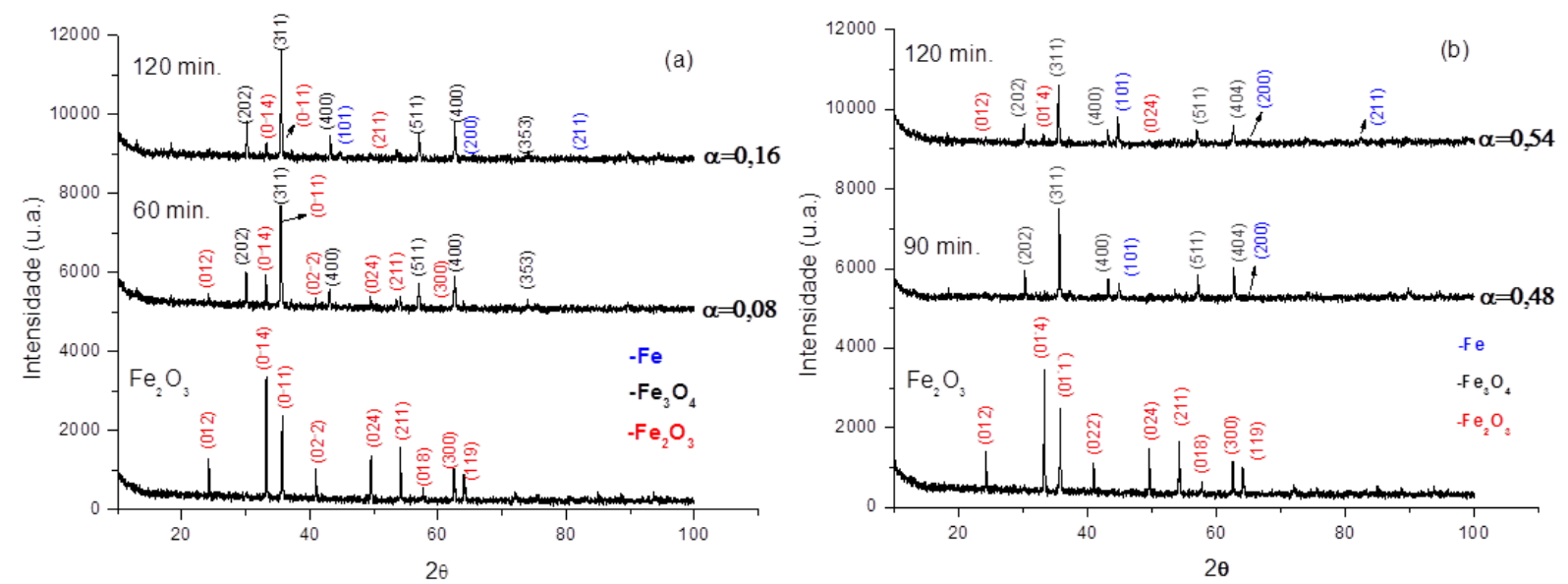

* Contribuição técnica ao 69 Congresso Anual da ABM - Internacional e ao 14ํㅡㄹ ENEMET - Encontro Nacional de Estudantes de Engenharia Metalúrgica, de Materiais e de Minas, 21 a 25 de julho de 2014, São Paulo, SP, Brasil. 


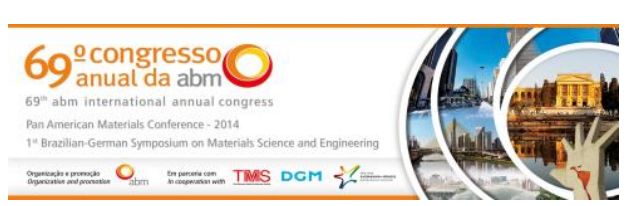

Figura 5. Evolução das fases presentes nos pós de hematita na redução por hidrogênio gasoso, a $360^{\circ} \mathrm{C}(\mathrm{a})$ e $400^{\circ} \mathrm{C}(\mathrm{b})$.

\subsection{Efeito da Adição de Argônio na redução de hematita por plasma frio de hidrogênio.}

O argônio (Ar) no estado de plasma apresenta alta intensidade de "sputtering" (pulverização catódica) [10,11]. Assim a pequena adição deste elemento objetiva-se criar mais defeitos sobre a superfície do óxido, para aumentar a área da interface plasma-óxido; além de auxiliar a redução pela ejeção de átomos de oxigênio e ferro do óxido [13]. O oxigênio ejetado reage e forma vapor d'agua e os átomos de ferro se depositam sobre a superfície novamente [11, 13]

A Figura 6 apresenta os resultados da redução de pós de hematita por plasma gerado pela mistura $2,5 \% \mathrm{Ar}+97,5 \% \mathrm{H}_{2}$ em comparação à redução por plasma de hidrogênio puro.

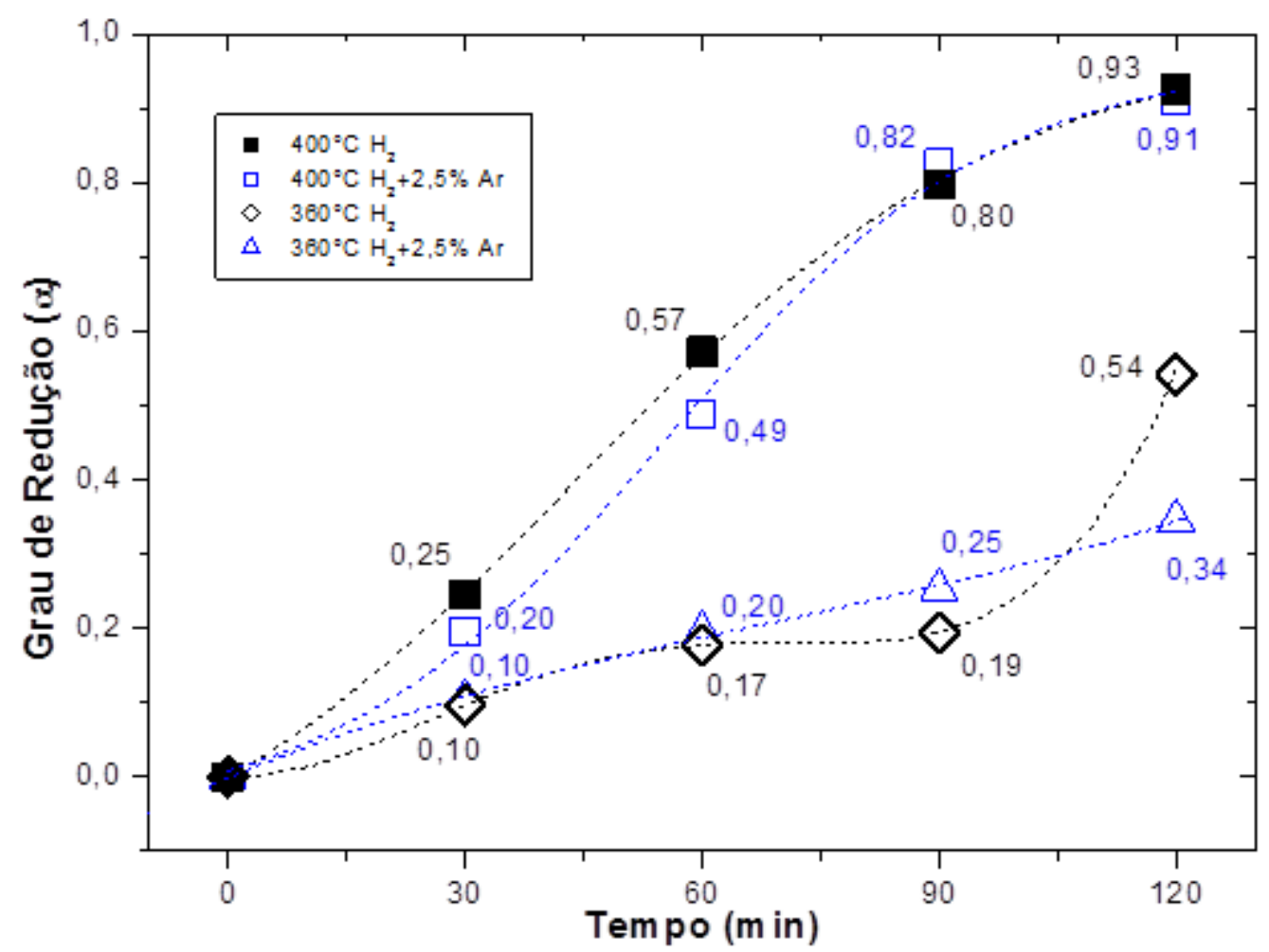

Figura 6. Grau de redução $(\alpha)$ em função do tempo (min.) dos ensaios de redução de hematita por plasma de $\mathrm{H}_{2}$ e plasma da mistura $2,5 \% \mathrm{Ar}+97,5 \% \mathrm{H}_{2}$.

Com essa mistura obtivemos redução dos pós de hematita equivalente aos experimentos de redução a plasma utilizando-se $\mathrm{H}_{2}$ puro a $400^{\circ} \mathrm{C}$ e 120 minutos ( $\alpha=0,91$ e $\alpha=0,93$ respectivamente), e para $T=360^{\circ} \mathrm{C}$ e 60 minutos $(\alpha=0,20$ e $\alpha=0,17$ respectivamente).

A $400^{\circ} \mathrm{C}$ os valores do grau de redução $(\alpha)$ da mistura são bem próximos da redução por plasma de hidrogênio puro a $400^{\circ} \mathrm{C}$. Estes pontos também se ajustam com boa aproximação a uma curva sigmoidal $\left(R^{2}=0,99727\right)$ porém a $360^{\circ} \mathrm{C}$ se ajustam melhor a uma curva de um polinômio de $3^{\circ}\left(R^{2}=0,99845\right)$.

Apesar de não terem sido alcançados melhores valores de $\alpha$, como se espera, a redução com a adição de $2,5 \%$ de argônio no plasma de hidrogênio, não apenas

* Contribuição técnica ao 69 Congresso Anual da ABM - Internacional e ao 14ํㅡㄹ ENEMET - Encontro Nacional de Estudantes de Engenharia Metalúrgica, de Materiais e de Minas, 21 a 25 de julho de 2014, São Paulo, SP, Brasil. 


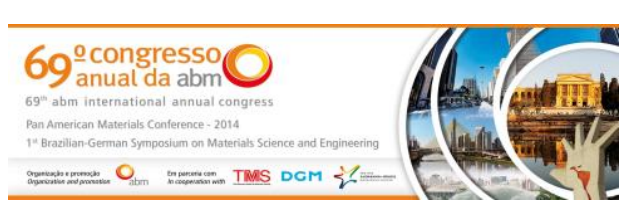

A adição de metano apresentou efeito deletério, pois foi necessário operar os ensaios com os maiores valores de $\tau$ on possíveis, a $360^{\circ} \mathrm{C}$ foi necessário uso de 130 $\mu \mathrm{s}$.

Além de desestabilizar o plasma, pois a descarga tornava-se instável com o aparecimento de arcos-elétricos a ponto de alguns ensaios terem sido abortados. Devido a estes efeitos, não foi possível manter os ensaios a $400^{\circ} \mathrm{C}$.

\section{CONCLUSÃO}

Redução a plasma de pós de hematita de elevada pureza por hidrogênio gasoso e por plasma frio com três misturas gasosas a saber: $\mathrm{H}_{2}, 2,5 \% \mathrm{Ar}+97,5 \% \mathrm{H}_{2}, 2,5 \%$ $\mathrm{CH}_{4}+97,5 \% \mathrm{H}_{2}$, nas temperaturas de $360^{\circ} \mathrm{C}$ e $400^{\circ} \mathrm{C}$ foram realizadas.

A redução dos pós de hematita por plasma frio de $\mathrm{H}_{2}$ mostrou-se mais eficiente em relação a redução por hidrogênio gasoso em todas as condições estudadas.

Foi obtida uma redução de $93 \%$ a partir da hematita a $400^{\circ} \mathrm{C}$ e 120 minutos, utilizando-se plasma de hidrogênio.

A adição de $2,5 \%$ de argônio $\left(2,5 \% \mathrm{Ar}+97,5 \% \mathrm{H}_{2}\right)$ alcançou um grau de redução de $91 \%$ a $400^{\circ} \mathrm{C}$ e 120 minutos, além de garantir melhor estabilidade do plasma e preservar o equipamento trabalhando com um valor de ton bem menor.

A adição de metano na mistura gasosa foi completamente prejudicial à redução além de prejudicar a estabilidade do plasma.

A redução dos pós de hematita por plasma seguiram-se duas etapas: $\mathrm{Fe}_{2} \mathrm{O}_{3} \rightarrow$ $\mathrm{Fe}_{3} \mathrm{O}_{4} \rightarrow \mathrm{Fe}(\alpha)$ ou $\mathrm{Fe}_{2} \mathrm{O}_{3} \rightarrow \mathrm{Fe}_{3} \mathrm{O}_{4}+\mathrm{Fe}_{(\alpha)} \rightarrow \mathrm{Fe}_{(\alpha)}$

A redução dos pós de hematita por hidrogênio gasoso nas condições de estudo seguiram as etapas: $\mathrm{Fe}_{2} \mathrm{O}_{3} \rightarrow \mathrm{Fe}_{2} \mathrm{O}_{3}+\mathrm{Fe}_{3} \mathrm{O}_{4} \rightarrow \mathrm{Fe}_{2} \mathrm{O}_{3}+\mathrm{Fe}_{3} \mathrm{O}_{4}+\mathrm{Fe}_{(\alpha)}$.

\section{Agradecimentos}

À Coordenação de Aperfeiçoamento de Pessoal de Nível Superior (CAPES) pela bolsa de mestrado concedida a Tiago Bristt Gonoring.

\section{REFERÊNCIAS}

1 Birat J.-P, Hanrot F, Danloy G. $\mathrm{CO}_{2}$ mitigation technologies in the steel industry: a benchmarking study based on process calculations. Stahl und Eisen, 2003; 123(9): 6972 .

2 Lampert $\mathrm{K}$, Ziebik $\mathrm{A}$, Stanek $\mathrm{W}$. Thermoeconomical analysis of $\mathrm{CO}_{2}$ removal from the Corex export gas and its integration with the blast-furnace assembly and metallurgical combined heat and power (CHP) plant. Energy, 2010; 35(2): 1188-1195.

3 Ghosh A, Chaterjee A. Ironmaking and steelmaking: theory and practice. PHI Learning Private Limited, New Delhi, 2008.

4 Hiebler $\mathrm{H}$, Plaul J. Hydrogen plasma smelting reduction - An option for steelmaking in the future. Metalurgija, 2004; 43(3): 155-162.

5 Rajput P, Bhoi B, Sahoo S, Paramguru KR, Mishra BK. Preliminary investigation into direct reduction of iron in low temperature hydrogen plasma. Ironmaking and Steelmaking, 2013; 40(1): 61-68.

6 Khina BB, Grinchuk PS. Modeling of iron scale reduction by methane conversion products in a plasma jet. I. Thermodynamic modeling. Journal of Engineering Physics and Thermophysics, 2010; 84(2): 280-286.

7 Ogorodnikova OV. Comparison of hydrogen gas-, atom-and ion-metal interactions. Journal of Nuclear Materials, 2000; 277(2): 130-142.

\footnotetext{
* Contribuição técnica ao $69^{\circ}$ Congresso Anual da ABM - Internacional e ao 14ํㅡㄹ ENEMET - Encontro Nacional de Estudantes de Engenharia Metalúrgica, de Materiais e de Minas, 21 a 25 de julho de 2014, São Paulo, SP, Brasil.
} 
